\title{
Survival outcomes for advanced kidney cancer patients in the era of targeted therapies
}

\author{
Arpit Rao ${ }^{1}$, Charles Wiggins ${ }^{2}$, Richard C. Lauer ${ }^{3}$ \\ ${ }^{1}$ Division of Hematology, Oncology \& Transplantation, University of Minnesota, Minnesota, MN, USA; ${ }^{2}$ Department of Internal Medicine, New \\ Mexico Tumor Registry, University of New Mexico, Albuquerque, NM, USA; ${ }^{3}$ Division of Hematology and Oncology, University of New Mexico \\ Comprehensive Cancer Center, Albuquerque, NM, USA \\ Contributions: (I) Conception and design: All authors; (II) Administrative support: A Rao; (III) Provision of study materials or patients: C Wiggins; \\ (IV) Collection and assembly of data: C Wiggins; (V) Data analysis and interpretation: A Rao, C Wiggins; (VI) Manuscript writing: All authors; (VII) \\ Final approval of manuscript: All authors. \\ Correspondence to: Arpit Rao, MD. Division of Hematology, Oncology \& Transplantation, University of Minnesota, Minnesota, MN 55455, USA. \\ Email: raoa@umn.edu.
}

Background: Advanced renal cell carcinoma (RCC) results in over 14,000 deaths each year in the United States alone. The therapeutic landscape for advanced RCC changed dramatically with the approval of tyrosine kinase inhibitors (TKI) between 2006 and 2012. A large-scale analysis of survival trends has not been performed in the TKI era to determine their impact on outcomes for advanced RCC patients.

Methods: The Surveillance, Epidemiology and End-Results (SEER) database was queried for adult patients with advanced RCC diagnosed between 2000 and 2013. Patients were divided into two cohorts based on the year of diagnosis-pre-TKI cohort [2000-2006] and TKI cohort [2007-2013]. Kaplan-Meier survival and multivariate Cox proportional hazards analyses were performed.

Results: A total of 14,976 patients were included in our study. Median age at diagnosis was 64 years (range, 18-89 years). Median (cancer-specific) overall survival was 10.0 months in the TKI cohort compared with 8.0 months in the pre-TKI cohort, corresponding to a $13 \%$ improvement in survival in the TKI area [hazard ratio (HR) for death 0.87 ; 95\% confidence interval (CI), 0.84-0.91, $\mathrm{P}<0.0001$ ]. Median survival was improved by 2 months for patients with clear-cell RCC histology [HR for death 0.86; 95\% CI, 0.84-0.91, $\mathrm{P}<0.0001]$. Patients with non-clear cell RCC had a 25\% higher risk of mortality compared with those with clear-cell RCC. Additionally, median survival for non-clear cell RCC patients was not statistically different between the two cohorts (HR for death 0.98; 95\% CI, 0.88-1.09, P=0.714). Subgroup analysis showed that elderly patients (age 71 years and above) had a $45 \%$ higher risk of death from advanced RCC compared with young patients (aged 18-50 years) [HR for death 1.45; 95\% CI, 1.36-1.54, P<0.0001]. Gender and racial disparities in outcomes were also noted with women having a $10 \%$ higher risk of death compared with men (HR for death 1.10; 95\% CI, 1.06-1.14, $\mathrm{P}<0.001$ ) and Black patients having a $15 \%$ higher risk of death compared with White patients (HR for death 1.15; 95\% CI, 1.08-1.23, $\mathrm{P}<0.0001$ ).

Conclusions: Our study provides a largest registry-based analysis of survival outcomes in the TKI era. In majority of patients, the survival has improved significantly with the advent of TKIs as standard of care therapy. Survival for patients with non-clear cell RCC is clearly worse than clear-cell RCC and does not appear to have changed with TKIs. Elderly patients, women, and Black patients appear to have worse outcomes and these disparities merit further investigation.

Keywords: Tyrosine kinase inhibitor (TKI); renal cell carcinoma (RCC); kidney; disparities; survival

Submitted Mar 21, 2018. Accepted for publication Apr 12, 2018.

doi: $10.21037 /$ atm.2018.04.44

View this article at: http://dx.doi.org/10.21037/atm.2018.04.44 


\section{Introduction}

Renal cell carcinoma (RCC) is the eighth most common malignancy in the United States, and advanced disease causes over 14,000 deaths each year. Approximately 16\% of patients present with distant (metastatic) disease, and 5 -year cancer-specific survival for these patients remains dismal at $11.2 \%(1-3)$. Clear-cell RCC is the most common histological subtype of RCC, found in approximately $75 \%$ of cases (3-5).

Between 2005 and 2012, several new agents targeting growth factor receptors on renal cancer cells-tyrosine kinase inhibitors (TKIs) - were approved by the US Food and Drug Administration (FDA) for use in metastatic RCC (6-8). These therapies dramatically altered the therapeutic landscape for RCC beginning in 2006.

While each of these TKIs have individually been shown to improve outcomes in metastatic RCC $(9,10)$, their impact as a whole remains to be determined. Also, because most of the targeted therapies were tested in patients with clear-cell RCC, the therapeutic efficacy of TKIs in patients with nonclear cell histologies of RCC remains unclear.

Racial disparities in survival have been reported in several cancers (11-13). Black patients with RCC have been shown to have poorer outcomes compared with White patients regardless of the stage at presentation. Socioeconomic factors and differences in prevalence of various histologies have been proposed as some of the reasons for these observations $(12,14)$. These disparities, however, have not been well-studied for patients with metastatic RCC in the era of TKIs.

\section{Methods}

The National Cancer Institute's Surveillance, Epidemiology and End-Results (SEER) database was queried for patients aged 18 years and older who were diagnosed with advanced (distant stage) RCC between January 1, 2000 and December 31, 2013 in the eighteen SEER registries (15). Analyses were restricted to histologically confirmed cases with available follow-up data. Patients diagnosed on or after January 1, 2014 were excluded due to non-availability of survival data.

The study period was divided into two 7-year intervals: 2000-2006 (the pre-TKI cohort) and 2007-2013 (the TKI cohort) based on the general consensus on 2007 as the year when TKIs started being commonly used in the US Data-retrieval for final analyses was performed in
January 2017.

\section{Statistical methods}

Variables used for stratification included patient's age at diagnosis, gender, race, histology, residence in metropolitan area, and socio-economic status (as defined by completion of high-school education). Age at diagnosis was divided into three categories (18-50, 51-70, and 71 years and older) based on known age distribution of the disease (1). Race was divided into (non-Hispanic) White, Black, and others (including Native American, Asian American, and Hispanic Whites).

The six most common RCC histologies were included: clear cell, papillary, medullary, chromophobe, cystassociated and sarcomatoid. Because of their significantly lower prevalence, the last five of these histologies were grouped into "non-clear cell histology" category for analysis. Any cases with non-standardized or mixed diagnoses codes (e.g., malignant renal neoplasm, clear cell with papillary features) were excluded from analyses.

Survival analyses including the 1, 2, and 3 years, and median (cancer-specific) survival were performed using the Kaplan-Meier method. Differences in cancer-specific survival by time period were assessed with the Log-Rank test. Multivariate Cox proportional hazards model was used for estimating hazard ratios (HR) for death and performing univariate and multivariate analyses. SAS statistical software package version $9.2\left({ }^{\circledR} \mathrm{SAS}\right.$ Institute, Inc.) was used for all analyses.

\section{Results}

A total of 15,015 patients with a new diagnosis of metastatic RCC were identified in the SEER database between 2000 and 2013, of which 14,976 patients met criteria for inclusion in the study. Median age at diagnosis was 64 years (range, 18-89 years). Demographic characteristics were balanced among the two cohorts (Table 1).

\section{Cancer-specific survival}

Median survival was 10.0 months in the TKI cohort compared with 8.0 months in the pre-TKI cohort, corresponding to a $13 \%$ improvement in survival in the TKI area [adjusted HR 0.87; 95\% confidence interval (CI), 0.84-0.91, $\mathrm{P}<0.0001]$. One-, 2-, and 3-year survival were 
Table 1 Baseline characteristics of patient population

\begin{tabular}{|c|c|c|c|}
\hline Characteristic & Category & \multicolumn{2}{|c|}{ Time period of diagnosis } \\
\hline \multirow[t]{3}{*}{ Age at diagnosis (years) } & $18-50$ & $1,100(15.97)$ & $1,186(14.66)$ \\
\hline & $51-70$ & $3,872(56.21)$ & $4,712(58.26)$ \\
\hline & $71+$ & $1,916(27.82)$ & $2,190(27.08)$ \\
\hline Sex & Female & 2,213 (32.13) & $2,510(31.03)$ \\
\hline \multirow[t]{3}{*}{ Race } & White & $5,817(84.45)$ & 6,707 (82.93) \\
\hline & Black & $680(9.87)$ & $790(9.77)$ \\
\hline & Other & $391(5.68)$ & $591(7.31)$ \\
\hline \multirow{3}{*}{ Histology categories } & Chromophobe & $60(0.87)$ & $68(0.84)$ \\
\hline & Sarcomatoid & $440(6.39)$ & $526(6.50)$ \\
\hline & Cyst-associated & $8(0.12)$ & $1(0.01)$ \\
\hline \multirow[t]{2}{*}{ Place of residence } & Metropolitan & $5,918(85.92)$ & $6,935(85.74)$ \\
\hline & Non-metropolitan & $970(14.08)$ & $1,153(14.26)$ \\
\hline \multirow{3}{*}{$\begin{array}{l}\text { Percent with less than high } \\
\text { school education (quartiles) (\%) }\end{array}$} & Q1: 00.94-10.58 & $1,973(28.64)$ & $2,206(27.27)$ \\
\hline & Q2: 10.59-15.55 & $1,585(23.01)$ & $1,731(21.40))$ \\
\hline & Q3: 15.56-25.75 & $1,720(24.97)$ & $2,027(25.06)$ \\
\hline
\end{tabular}

better in the TKI cohort compared with pre-TKI cohort (Figure 1).

\section{Survival by bistology}

For patients with clear-cell RCC, the median survival was 10.0 months in the TKI cohort compared with 8.0 months in the pre-TKI cohort, corresponding to a $14 \%$ improvement in survival in the TKI (HR for death 0.86 ; 95\% CI, 0.84-0.91, $\mathrm{P}<0.0001$ ) (Figure 2).

For those with non-clear cell RCC, the median survival was 7.0 months in both cohorts, a statistically insignificant difference (HR for death 0.98; 95\% CI, 0.88-1.09, $\mathrm{P}=0.714$ ) (Figure 3).

Thus, patients with non-clear cell RCC had a $25 \%$ higher risk of mortality compared with those with clear-cell RCC (HR for death 1.25; 95\% CI, 1.17-1.32, $\mathrm{P}<0.0001$ ). This difference persisted despite adjustment for the year of diagnosis, suggesting that patients diagnosed in the TKI era continue to have poorer outcomes.

\section{Survival by age at diagnosis}

Median survival improved across all ages in the TKI cohort. For young patients (ages, 18 to 50 years), the median survival was 13.0 months in TKI cohort compared with 8.0 months in pre-TKI cohort $(\mathrm{P}<0.0001)$ in the TKI cohort. For middle-aged patients (ages, 51-70 years), median survival was 11.0 months in the TKI cohort and 9.0 months in pre-TKI cohort $(\mathrm{P}<0.0001)$. For elderly patients (ages 71 and above), median survival was 6.0 months in the TKI cohort and 5.0 months in pre-TKI cohort $(\mathrm{P}=0.002)$.

Additionally, compared with young patients, middle-aged patients had a $7 \%$ higher risk of death from advanced RCC (HR for death 1.07 ; 95\% CI, 1.02-1.13, $\mathrm{P}=0.013$ ). Elderly 


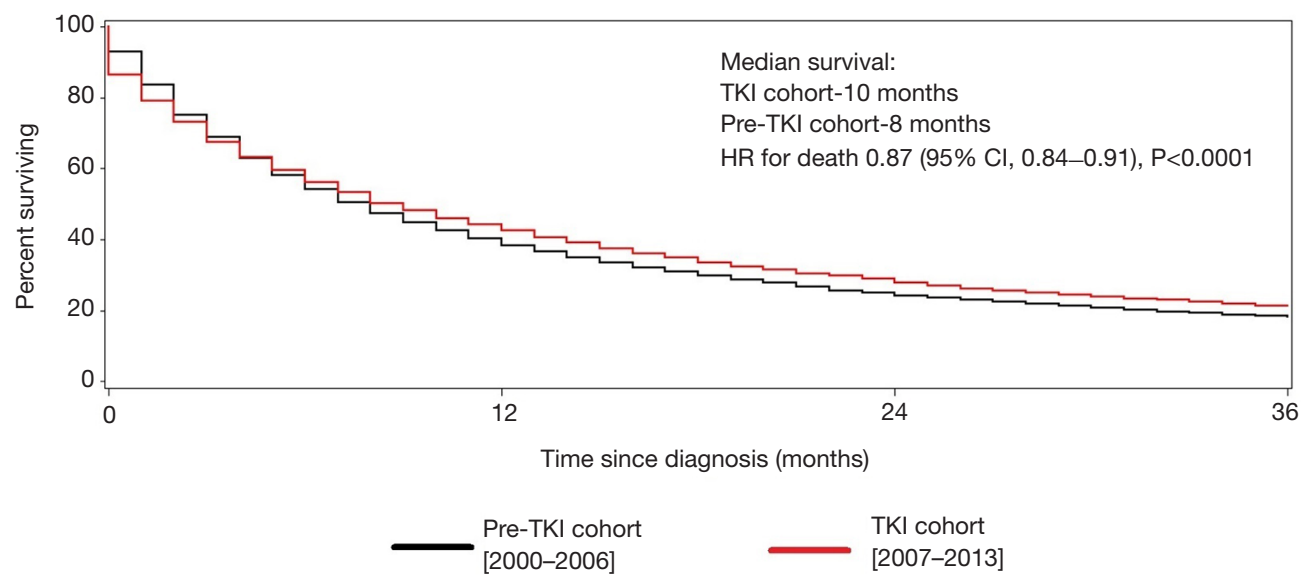

Figure 1 Cancer-specific survival (all histologies). Kaplan-Meier curves for the (cancer-specific) survival for all histologies of renal cell carcinoma. Pre-TKI cohort is represented by the black line and the TKI cohort by the red line. TKI, tyrosine kinase inhibitor; CI, confidence interval; HR, hazard ratio.

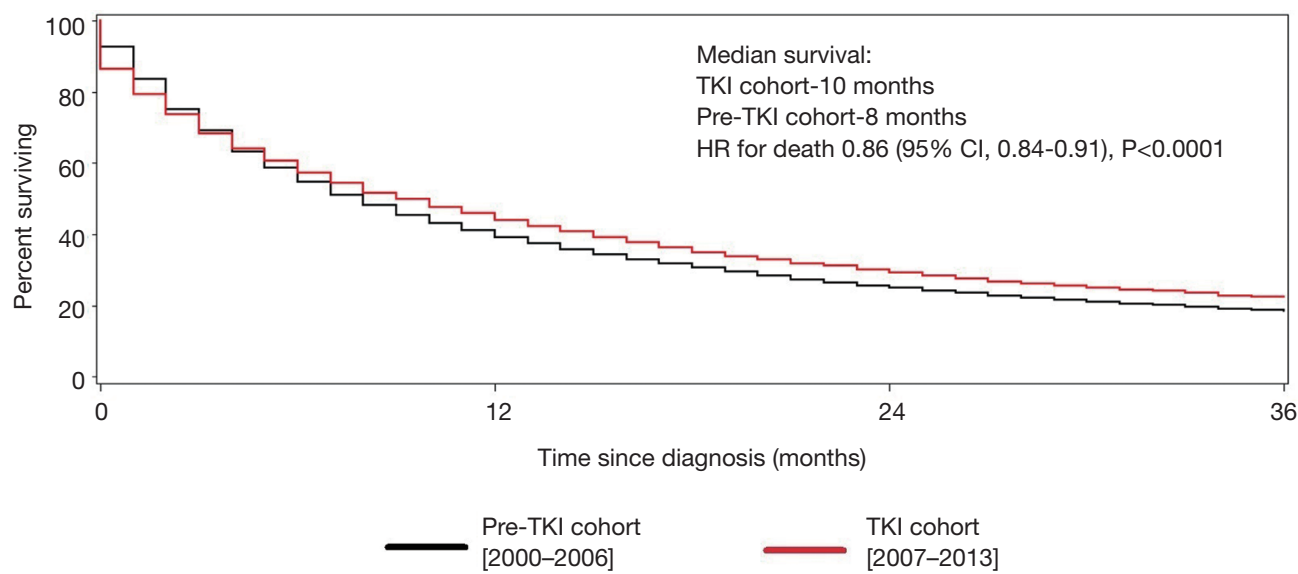

Figure 2 Cancer-specific survival (clear-cell histology). Kaplan-Meier curves for the (cancer-specific) survival for patients with clear-cell renal cell carcinoma. Pre-TKI cohort is represented by the black line and the TKI cohort by the red line. TKI, tyrosine kinase inhibitor; CI, confidence interval; HR, hazard ratio.

patients fared much worse, with a $45 \%$ higher risk of death from advanced RCC compared with young patients (HR for death 1.45; 95\% CI, 1.36-1.54, $\mathrm{P}<0.0001)$.

\section{Survival by gender}

For males, the median survival was 10.0 months in TKI cohort and 8.0 months in the pre-TKI cohort $(\mathrm{P}<0.0001)$. For females, the median survival was 9.0 months in TKI cohort and 7.0 months in pre-TKI cohort $(\mathrm{P}<0.0001)$.

Women were found to have a $10 \%$ higher risk of death from advanced RCC compared with males (HR for death
1.10; 95\% CI, 1.06-1.14, $\mathrm{P}<0.001)$ despite adjustment for covariates including year of diagnosis (i.e., in both pre-TKI and TKI cohorts) and histology of RCC.

\section{Survival by race}

In White patients, the median survival was 13.0 months in TKI cohort and 8.0 months in pre-TKI cohort $(\mathrm{P}<0.0001)$. For Black patients, median survival was 7.0 months in TKI cohort and 6.0 months in pre-TKI cohort, a difference that was statistically non-significant $(\mathrm{P}=0.349)$.

Furthermore, compared with White patients, Black 


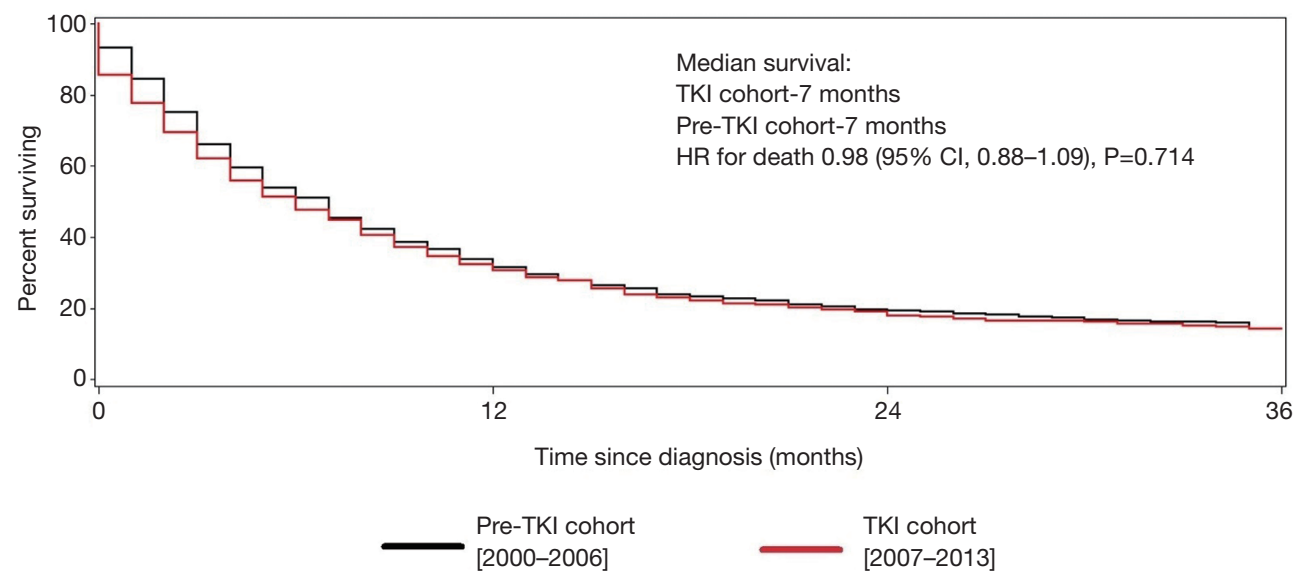

Figure 3 Cancer-specific survival (non-clear cell histology). Kaplan-Meier curves for the (cancer-specific) survival for patients with nonclear cell renal cell carcinoma. Pre-TKI cohort is represented by the black line and the TKI cohort by the red line. TKI, tyrosine kinase inhibitor; CI, confidence interval; HR, hazard ratio.

Table 2 Survival characteristics based on the socio-economic status

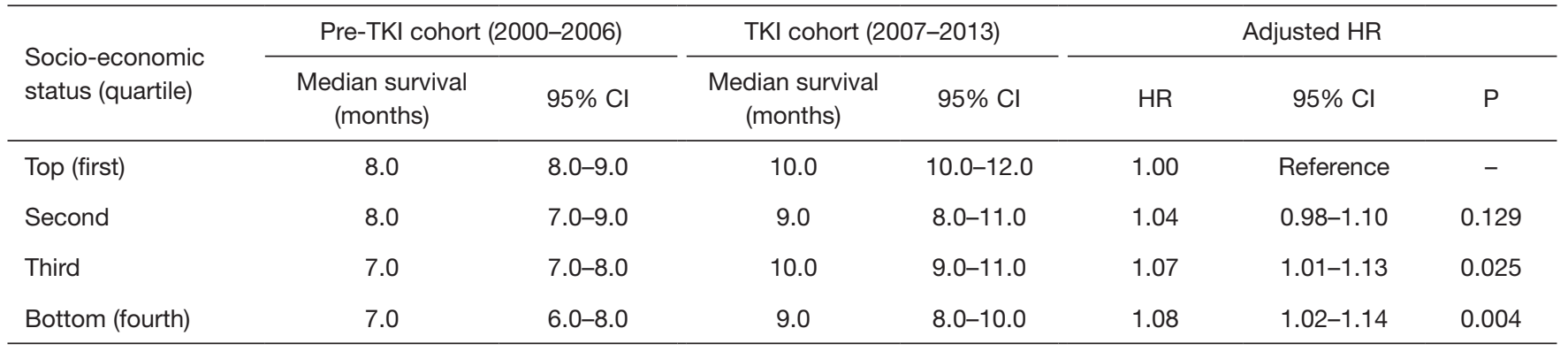

$\mathrm{HR}$, hazard ratio; $\mathrm{Cl}$, confidence interval; TKI, tyrosine kinase inhibitor.

patients had approximately $15 \%$ higher risk of death from advanced RCC (unadjusted HR for death 1.19; 95\% CI, 1.08-1.18, $\mathrm{P}<0.0001)$. This difference persisted despite adjustment for covariates including age, gender, histology, and socio-economic status (adjusted HR for death 1.15; $95 \%$ CI, $1.08-1.23, \mathrm{P}<0.0001)$.

\section{Survival by location}

Improvement in survival was noted for patients residing in metropolitan and non-metropolitan areas. For patients residing in a metropolitan area, the median survival was 10.0 months in TKI cohort compared with 8.0 months in pre-TKI cohort $(\mathrm{P}<0.0001)$. For patients residing in a nonmetropolitan area, the median survival was 9.0 months in TKI cohort compared with 8.0 months (95\% CI, 7.0-9.0) in pre-TKI cohort $(\mathrm{P}=0.022)$.
No significant differences in survival were noted between patients living in metropolitan versus non-metropolitan areas (HR for death 1.00; 95\% CI, 0.95-1.06, $\mathrm{P}=0.899$ ).

\section{Survival by socio-economic status (SES)}

Compared with the patients in highest (first) quartile, patients in the third and fourth quartile (signifying a lower SES) had about $8 \%$ higher likelihood of mortality from RCC (HR for death 1.08; 95\% CI, 1.02-1.14, P=0.004) (Table 2).

\section{Discussion}

Our study provides a detailed insight into the survival outcomes in the TKI era for patients with advanced RCC in the United States. We are pleased to report that the 
cancer-specific survival has improved significantly in the TKI era, and this difference appears to be primarily driven by patients with clear-cell histology. However, the survival does not appear to have improved for patients with nonclear cell histologies. Difference in disease biology may provide a sufficient explanation, but the extent to which the patients with non-clear cell histologies were offered TKI remains unknown.

We also found a significantly higher risk of death in elderly patients, which persisted despite adjustment for covariates. This stark difference in the risk of death was also similar in pre-TKI and TKI cohorts, suggesting that receipt of TKI is very unlikely to solely explain the poorer survival of elderly patients with RCC. Ultimately however, it is very likely that a combination of multiple factors including comorbidities, disease biology, functional reserve, psychosocial factors, and treatment receipt and tolerance (especially for second line therapy) contributed to the noted difference in outcomes (16). A SEERMedicare or payer-database analysis may help validate our findings by resolving the impact of receipt of therapy as a potential confounder.

A surprising finding was a $10 \%$ higher rate of mortality in females compared with males. This could represent a statistically significant albeit clinically insignificant finding. However, gender-specific differences in outcomes have not received much attention in RCC, and the cause of these differences merits further investigation.

Our study also reaffirmed the reported racial disparities in patients with RCC. Socio-economic disparity and a higher prevalence of papillary histology had been proposed as possible reasons for this disparity $(12,14,17-19)$. Thus, additional research is warranted to confirm our findings and inform policies to help decrease racial disparities in outcomes.

\section{Limitations}

Our study shares its limitations with other large population database-based studies. These include potential errors stemming from inadequate quality control of collected data-for example, a significant number of patients with incorrect or mixed diagnoses codes were excluded from our study, which may have adversely affected some estimates for non-clear cell RCC histologies. Due to lack of correlative clinical data, several known and some potentially unknown confounders (e.g., nephrectomy status, IMDC riskstratification, the location and total burden of metastatic disease, and comorbidities) could have biased the analyses. Finally, we were unable to analyze actual TKI use, which could have helped identify potential reasons for poorer outcomes.

\section{Conclusions}

Our study clearly demonstrates the improvement in survival for patients with advanced RCC in the TKI era. This improvement appears to be primarily driven by patients with the clear cell histologic subtype of RCC. This finding lends additional support to the belief of genitourinary medical oncology community that effective therapies specifically for patients with non-clear histologies are urgently needed. Our group is planning a SEER-Medicare analysis to evaluate the cause(s) behind the starkly inferior outcomes in elderly patients with advanced RCC. Finally, the gender and race-based disparities noted in our study warrant further investigation.

\section{Acknowledgements}

None.

\section{Footnote}

Conflicts of Interest: The authors have no conflicts of interest to declare.

\section{References}

1. Cancer of the Kidney and Renal Pelvis_-SEER Stat Fact Sheets. Available online: http://seer.cancer.gov/statfacts/ html/kidrp.html

2. Chow WH, Dong LM, Devesa SS. Epidemiology and risk factors for kidney cancer. Nat Rev Urol 2010;7:245-57.

3. Ljungberg B, Campbell SC, Choi HY, et al. The epidemiology of renal cell carcinoma. Eur Urol 2011;60:615-21.

4. Cheville JC, Lohse CM, Zincke H, et al. Comparisons of outcome and prognostic features among histologic subtypes of renal cell carcinoma. Am J Surg Pathol 2003;27:612-24.

5. Shuch B, Hofmann JN, Merino MJ, et al. Pathologic validation of renal cell carcinoma histology in the Surveillance, Epidemiology, and End Results program. Urol Oncol 2014;32:23.e9-13.

6. Vogelzang NJ. Another step toward the cure of metastatic 
renal cell carcinoma? J Clin Oncol 2010;28:5017-9.

7. Drugs Approved for Kidney (Renal Cell) Cancer. Available online: http://www.cancer.gov/about-cancer/treatment/ drugs/kidney

8. Logan JE, Rampersaud EN, Sonn GA, et al. Systemic therapy for metastatic renal cell carcinoma: a review and update. Rev Urol 2012;14:65-78.

9. Harshman LC, Xie W, Bjarnason GA, et al. Conditional survival of patients with metastatic renal-cell carcinoma treated with VEGF-targeted therapy: a population-based study. Lancet Oncol 2012;13:927-35.

10. Pal SK, Nelson RA, Vogelzang N. Disease-specific survival in de novo metastatic renal cell carcinoma in the cytokine and targeted therapy era. PLoS One 2013;8:e63341.

11. National Cancer Institute Cancer Health Disparities. Available online: https://www.cancer.gov/about-nci/ organization/crchd/cancer-health-disparities-fact-sheet

12. Chow WH, Shuch B, Linehan WM, et al. Racial disparity in renal cell carcinoma patient survival according to demographic and clinical characteristics. Cancer 2013;119:388-94.

13. Doubeni CA, Rustgi A. Racial Disparities in Colorectal

Cite this article as: Rao A, Wiggins C, Lauer RC. Survival outcomes for advanced kidney cancer patients in the era of targeted therapies. Ann Transl Med 2018;6(9):165. doi: 10.21037/ atm.2018.04.44
Cancer Survival: Is Elimination of Variation in Care the Cure? J Natl Cancer Inst 2015;107.

14. Olshan AF, Kuo TM, Meyer AM, et al. Racial difference in histologic subtype of renal cell carcinoma. Cancer Med 2013;2:744-9.

15. Surveillance, Epidemiology, and End Results (SEER) Program Populations (2001-2014). Available online: www. seer.cancer.gov/popdata

16. Berger NA, Savvides P, Koroukian SM, et al. Cancer in the elderly. Trans Am Clin Climatol Assoc 2006;117:147-55; discussion 155-6.

17. Lin J, Zahm SH, Shriver CD, et al. Survival among Black and White patients with renal cell carcinoma in an equal-access health care system. Cancer Causes Control 2015;26:1019-26.

18. Lipworth L, Tarone RE, McLaughlin JK. Renal cell cancer among African Americans: an epidemiologic review. BMC Cancer 2011;11:133.

19. Sankin A, Cohen J, Wang H, et al. Rate of renal cell carcinoma subtypes in different races. Int Braz J Urol 2011;37:29-32; discussion 33-4. 